\title{
Generalización de la integral clásica a integral de orden arbitrario
}

\author{
Generalization of the classic integral to arbitrary order integral
}

${ }^{1}$ Jhony Alfonso Chávez Delgado ${ }^{2}$ Blanca Mayumi Gomez Challo

\section{RESUMEN}

El propósito de este artículo de investigación es demostrar la generalización de la integral de orden entero de NewtonLeibniz al operador de integración de orden arbitrario de Riemann-Liouville sobre un intervalo cerrado o finito que en la actualidad son atractivo a investigaciones de otras ciencias puras y aplicadas como la matemática, física, química, biología, etc. En tal sentido, se presentaron la teoría básica de las diversas aproximaciones de la integral de orden entero, utilizando la función Gamma y la fórmula de Cauchy; las cuales sirvieron de base para la definición del operador de integración a partir de la n-ésima integral iterada ordinaria de una función definida recursivamente. Así mismo, se emplearon los métodos lógicos inductivo - deductivo para las demostraciones de la fórmula de Cauchy. Finalmente, se aplicó esta teoría al análisis de la integral arbitraria de la función constante, potencia y logarítmica; contrastándolo con los ejemplos de la definición de Riemann-Liouville.

Palabras clave: integral arbitraria, integral arbitraria de la potencia, integral arbitraria de la función constante, integral arbitraria logarítmica.

\begin{abstract}
The purpose of this research article is to demonstrate the generalization of the Newton-Leibniz whole-order integral to the Riemann-Liouville arbitrary order integration operator over a closed or finite interval that is currently attractive to investigations of other pure sciences and applied as mathematics, physics, chemistry, biology, etc. In that sense, the basic theory of the various approximations of the whole order integral was presented, using the Gamma function and the Cauchy formula; this served as the basis for the definition of the integration operator from the ordinary nth iterated integral of a recursively defined function. Likewise, the inductive-deductive logical methods were used for the demonstrations of the Cauchy formula. Finally, this theory was applied to the analysis of the arbitrary integral of the constant, power and logarithmic function; contrasting it with the examples of the definition of Riemann-Liouville.
\end{abstract}

Keywords: arbitrary integral, arbitrary integral of power, arbitrary integral of constant function, logarithmic arbitrary integral.

\section{INTRODUCCIÓN}

El cálculo de la integral de Newton y Leibniz como rama del análisis matemático, a través del tiempo, ha sido estudiado por reconocidos matemáticos, quienes han contribuido al desarrollo de lo que hoy conocemos como integral arbitraria y que tiene interés en las órdenes de todo operador de integración. Entre ellos destacamos a Euler (1738), Liouville (1832) y Riemann (1876). Asimismo, Bertram (1975); Miller y Ross (1993); Samko, Kilbas Marichev (1993); Bonilla, Kilbas y Trujillo (2003); Arafet, Domínguez y Chang (2008); Santanu (2011); Ortigueira.

Uno de los propósitos de este artículo es establecer la generalización de la integral de orden entero de Newton-Leibniz a orden arbitrario de Riemann-
Liouville, utilizando como base la función gamma y la fórmula de Cauchy para desarrollar la integral, debido a que ,en el estudio del cálculo integral los estudiantes de las ciencias formales solucionan integrales enteras de las funciones potencia, logarítmica, etc. algunos cuestionan por que los operadores de integración de la función logarítmica y potencia no han de ser un numero arbitrario.

Así mismo, la generalización de la integral de orden ordinario a orden arbitrario se justifica debida principalmente a la gran importancia para el desarrollo del cálculo arbitrario, porque con la integral también se explican el uso de la derivada arbitraria y las ecuaciones diferenciales ordinarias fraccionarias. A pesar de la gran cantidad de publicaciones en este campo, aun se necesita formalizar y ordenar los

'Universidad Nacional Jorge Basadre Grohmann. Tacna-Perú. E-mail: jchavezd@unjbg.edu.pe

'Universidad Nacional Jorge Basadre Grohmann, Tacna-Perú. E-mail: mayumigomez177005@gmail.com 
conceptos, proposiciones y ejemplificaciones de la integral y la derivada arbitraria, para hacerla más atractivas y de fácil acceso a las investigaciones de otras ciencias puras y aplicadas como la matemática, física, química, biología y economía.

\section{FUNCIÓN DE EULER:}

\section{Definición (Función Euleriana)}

Sea $x>0$. A la función $\Gamma: \mathrm{R}^{+} \rightarrow \mathrm{R}$ definida por $\Gamma(x)=\int_{0}^{+\infty} e^{-t} t^{x-1} d t$ se le denomina función Gamma de Euler.

\section{Propiedades de la función Gamma de Euler:}

Sea $x>0$,entonces se verifica las siguientes propiedades:

$\Gamma(1)=1$

$\Gamma\left(\frac{1}{2}\right)=\sqrt{\pi}$

$\Gamma(x+1)=x \Gamma(x), x>0$

$\mathrm{Si} \mathrm{n}$ es un entero no negativo, entonces $\Gamma(x+1)=x$ !

$\Gamma\left(n+\frac{1}{2}\right)=\frac{1 \times 3 \times 5 \times \ldots \times(2 n-1) \sqrt{\pi}}{2^{n}}$

$\mathrm{Si} x$ es un entero positivo: $\Gamma\left(x+\frac{1}{2}\right)=\frac{(2 x) ! \sqrt{\pi}}{2^{2 x} x !}$

Bell (1968), y Levedev (1992)

Tabla1.Valores de la función Gamma

\begin{tabular}{|c|c|}
\hline$\Gamma(x)=\int_{0}^{+\infty} e^{-t} t^{x-1} d t, x>0$ \\
\hline$\Gamma\left(\frac{1}{2}\right)$ & 1.7725 \\
\hline$\Gamma\left(\frac{3}{2}\right)$ & 0.8862 \\
\hline$\Gamma\left(\frac{3}{4}\right)$ & 1.2254 \\
\hline$\Gamma\left(\frac{5}{6}\right)$ & 1.1288 \\
\hline$\Gamma\left(\frac{7}{6}\right)$ & 0.9277 \\
\hline$\Gamma\left(\frac{1}{3}\right)$ & 2.6789 \\
\hline$\Gamma\left(\frac{4}{3}\right)$ & 0.8930 \\
\hline$\Gamma\left(\frac{11}{6}\right)$ & 0.9407 \\
\hline$\Gamma\left(\frac{19}{6}\right)$ & 2.3451 \\
\hline
\end{tabular}

Fuente: Elaboración propia con Bell, (1968) y Levedev, (1972)

Definición (Función Beta)

La función Beta es una función $\mathrm{B}: \mathrm{R} \times \mathrm{R} \rightarrow \mathrm{R}$

tal que $\mathrm{B}(x, y)=\int_{0}^{1} s^{x-1}(1-s)^{y-1} d s$; $x>0, y>0$ Para establecer la relación entre la función gamma y beta se consideran las siguientes propiedades: 
Si $x>0, y>0$, entonces

$$
\mathrm{B}(x, y)=\frac{\Gamma(x) \cdot \Gamma(y)}{\Gamma(x+y)}
$$

Si $x>0, y>0$, entonces

$$
\begin{gathered}
\mathrm{B}(x, y)=\mathrm{B}(y, x) \\
\text { Si } m<x<m+1 \text {, con } x=\alpha+m \\
\text { y } 0<\alpha<1 \text {, entonces } \\
\Gamma(x) \cdot \Gamma(1-x)=\frac{\pi}{\operatorname{sen}(\pi \cdot x)}
\end{gathered}
$$

Si $2 x \in Z_{0}^{+}$, entonces

$$
\Gamma(x) \cdot \Gamma\left(1+\frac{1}{2}\right)=2^{1-2 x} \cdot \Gamma(2 x) \sqrt{\pi}
$$

(Stein \& Shakarchi, 2003)

\section{Definición (Función $\boldsymbol{\psi}$ de Euler)}

La función $P S i$ es la función $\psi: \mathrm{R} \rightarrow \mathrm{R}$ definida como $\psi(z)=\frac{1}{\Gamma(z)} \frac{d}{d z} \Gamma(z), z>0$ , también se le conoce como la función digamma.

\section{Propiedades de la función $P s i$ :}

$\psi(z+1)=\frac{1}{\mathrm{Z}}+\psi(z), z>0$

$-\psi(1)=\Gamma^{\prime}(1)=\gamma$, donde

$\gamma=0.577215664901532860606512090$

$$
\gamma=\lim _{m \rightarrow \infty}\left\{\frac{1}{1}+\frac{1}{2}+\ldots+\frac{1}{m}-\ln (m)\right\}
$$

Si $\alpha>-1 \mathrm{y} \beta>-1$ entonces

$$
\Gamma(z)=\int_{0}^{1} \frac{x^{\alpha}-x^{\beta}}{1-x} d z=\psi(\beta+1)-\psi(\alpha+1)
$$

(Chaudhry \& Zubair ,2002).

Tabla 2. Valores de la función $P s i \quad$ de Euler

\begin{tabular}{|c|c|}
\hline$\psi(z)=\frac{1}{\Gamma(z)} \frac{d}{d z} \Gamma(z), z>0$ \\
\hline$\psi(1)$ & $-\gamma$ \\
\hline$\psi(n+1)$ & $-\gamma+1+\frac{1}{2}+\frac{1}{3}+\ldots+\frac{1}{n}$ \\
\hline$\psi\left(\frac{1}{2}\right)$ & $-\gamma-2 \ln (2)$. \\
\hline$\psi\left(\frac{1}{3}\right)$ & $-\gamma-\frac{\pi}{2 \sqrt{3}}-\frac{3}{2} \ln (3)$ \\
\hline$\psi\left(\frac{1}{4}\right)$ & $-\gamma-\frac{\pi}{2}-3 \ln (2)$ \\
\hline$\psi\left(\frac{2}{3}\right)$ & $-\gamma+\frac{\pi}{12 \sqrt{3}}-\frac{3}{2} \ln (3)$ \\
\hline
\end{tabular}

Fuente: Fuente elaboración propia con Chaudhry \& Zubair (2002)

\section{Definición (Función Gamma incompleta)}

La función Gamma incompleta es

$$
\gamma(\mathrm{V}, x)=\int_{0}^{x} t^{\mathrm{V}-1} e^{-t} d t, t>0
$$

Miller (1993)

\section{Espacio $L^{p}([\boldsymbol{a}, \boldsymbol{b}])$}

Sea $p \geq 1$.El espacio de funciones integrables en $[a, b]$, denotado por $L^{p}([a, b])$, se define:

$$
\begin{aligned}
& L^{p}([a, b])=\left\{\begin{array}{l}
f:[a, b] \rightarrow \mathrm{R} / f \text { es medible en } \\
\{a, b] \mathrm{y} \int_{a}^{b}|f(x)|^{p} d x<\infty
\end{array}\right\} \\
& { }_{a} I_{x}^{\alpha}(x-a)^{\beta}=\frac{\Gamma(\beta+1)}{\Gamma(\alpha+\beta+1)}(x-a)^{\alpha+\beta}
\end{aligned}
$$

(Ross, 1977) 


\section{MATERIALES Y MÉTODOS}

Nuestro artículo de investigación corresponde a las ciencias formales y considera como material de estudio las funciones reales de una variable real definidas en los espacios $L^{1}([a, b])$.

En este artículo se empleó el método inductivo deductivo para la generalización de la integral clásica a integral de orden arbitrario de las funciones constante, potencia y logaritmo. Se contrastó las demostraciones usando ejemplos elementales.

\section{RESULTADOS}

\section{La Integral arbitraria}

Sea $\alpha \in \mathrm{R}^{+}$e $f$ una función integrable en $L^{1}[a, b]$. Entonces $\forall x \in[a, b]$ ${ }_{a} I_{x}^{\alpha} f(x)=\frac{1}{\Gamma(\alpha)} \int_{a}^{x}(x-s)^{\alpha-1} f(s) d s$

(Formula de Cauchy)

\section{Integral arbitraria de una función constante}

Si $f: \mathrm{R} \rightarrow \mathrm{R}$ es una función tal que $f(x)=k$ entonces

$$
\left({ }_{a} I_{x}^{\alpha} f\right)(x)=\frac{k}{\Gamma(\alpha+1)}(x-a)^{\alpha}
$$

Integral arbitraria de la función potencia

Si $f(x)=(x-a)^{\beta}, \beta>0, x>a, \alpha>0$ entonces

Miller \& Ross (1993)

\section{Integral arbitraria de la función logaritmo}

Si $f(x)=\ln (x-a)$ tal que $x>a, \alpha>0$ entonces

$$
{ }_{a} I_{x}^{\alpha} \ln (x-a)=\frac{(x-a)^{\alpha}}{\Gamma(\alpha+1)}[\ln (x-a)-\gamma-\psi(\alpha] 1)
$$

Miller \& Ross (1993)

\section{DISCUSIÓN}

Definición (Fubbini) Si $f:[a, b] \rightarrow \mathbb{R}$ es una función continua en $[a, b], b>x$, entonces

$$
\int_{a}^{x} d x_{1} \int_{a}^{x_{1}} f(t) d t=\int_{a}^{x} d t \int_{a}^{x} f(t) d x_{1}
$$

Miller \& Ross (1993)

\section{La Integral arbitraria:}

Proposición (Fórmula de Cauchy)

Sea $n \in \mathrm{N}$ y $f \in L^{1}[a, b]$.Entonces la enésima integral está dado por

$$
{ }_{a} I_{x}^{n} f(x)=\frac{1}{\Gamma(n)} \int_{a}^{x}(x-t)^{n-1} f(t) d t, t>a, n \in \mathrm{N}
$$

\section{Prueba}

Sea $f \in L^{1}[a, b]$. Luego, definimos para $x \in[a, b]$ la función $f$ con límite de integración inferior $a=0$ :

$$
{ }_{0} I_{x}^{1} f(x)=\int_{0}^{x} f(t) d t, t>a, n \in \mathrm{N}
$$

Para dos integrales se tiene:

$$
{ }_{0} I_{x}^{2} f(x)=\int_{0}^{x} d x_{1} \int_{0}^{x_{1}} f(t) d t
$$

Por la definición dada, podemos intercambiar el orden de las integrales en la integral doble

$$
{ }_{0} I_{x}^{n} f(x)=\frac{1}{\Gamma(n)} \int_{a}^{x}(x-t)^{n-1} f(t) d t, t>a, n \in \mathrm{N}
$$

Supongamos que para $n=k$ se cumple (1.1)

$$
\begin{aligned}
&{ }_{a} I_{x}^{k} f(x)=\frac{1}{\Gamma(k)} \int_{a}^{x}(x-t)^{k-1} f(t) d t \\
&{ }_{0} I_{x}^{2} f(x)=\int_{0}^{x} d t \int_{0}^{x} f(t) d x \\
&=\int_{0}^{x} f(t) d t \int_{t}^{x} d x_{1} \\
&=\int_{0}^{x}(x-t) f(t) d t
\end{aligned}
$$


Ahora consideremos tres integrales:

$$
\begin{aligned}
{ }_{0} I_{x}^{3} f(x) & =\int_{0}^{x} d x_{1} \int_{0}^{x_{1}} d x_{2} \int_{0}^{x_{2}} f(t) d t \\
& =\int_{0}^{x} d x_{1}\left[\int_{0}^{x_{1}} d x_{2} \int_{0}^{x_{2}} f(t) d t\right] \\
& =\int_{0}^{x} f(t) d t \int_{t}^{x}\left(x_{1}-t\right) d x_{1} \\
& =\int_{0}^{x} \frac{(x-t)^{2}}{2} f(t) d t \\
& =\frac{1}{1.2} \int_{0}^{x} f(t)[x-t]^{2} d t
\end{aligned}
$$

De lo anterior se deduce que para cuatro integrales se tiene:

$$
{ }_{0} I_{x}^{4} f(x)=\frac{1}{1.2 .3} \int_{0}^{x} f(t)[x-t]^{3} d t
$$

, y para $n$ integrales:

$$
\begin{aligned}
& { }_{0} I_{x}^{n} f(x)=\int_{0}^{x} d x_{1} \int_{0}^{x_{1}} d x_{2} \ldots \int_{0}^{n-2} d x \int_{n}^{n-1} f(t) d t \\
& { }_{0} I_{x}^{n} f(x)=\frac{1}{\Gamma(n)} \int_{a}^{x} f(t)[x-t]^{n-1} f(t) d t, t>a, n \in \mathrm{N}
\end{aligned}
$$

Además, como el límite inferior de la integral y arbitrario entonces se obtiene:

Entonces se tiene para $n=k+1$ :

$$
\begin{aligned}
& { }_{a} I_{x}^{k+1} f(x)=\int_{a}^{t} \frac{1}{\Gamma(k)} \int_{a}^{x}(t-r)^{k-1} f(r) d r d t \\
& { }_{a} I_{x}^{k+1} f(x)=\frac{1}{k \cdot \Gamma(k)} \int_{a}^{t}(t-r)^{k} f(r) d r \\
& { }_{a} I_{x}^{k+1} f(x)=\frac{1}{\Gamma(k+1)} \int_{a}^{t}(t-r)^{k} f(r) d r
\end{aligned}
$$

La ecuación (1.1) es conocida como la fórmula de Cauchy y puede extenderse para cualquier $\alpha \in \mathbb{R}^{+}$, en la siguiente definición:

\section{Definición (Operador de integración de orden arbitrario Riemann-Liouville)}

Sea $\alpha \in \mathbb{R}^{+}$.El operador de integración de orden arbitrario definido por ${ }_{a} I_{x}^{\alpha}$, es del tipo RiemannLiouville de orden $\alpha$ de una función $f \in L^{1}[a, b]$.

$$
{ }_{a} I_{x}^{\alpha} f(x)=\frac{1}{\Gamma(\alpha)} \int_{a}^{\alpha}[x-s]^{\alpha-1} f(s) d s
$$

\section{Ejemplo (Operador de integración arbitrario)}

De la definición de Riemann-Liouville calcule la integral arbitraria de orden $\alpha=\frac{3}{2}$, en la función cuya regla de correspondencia es $f(x)=2$

\section{Solución}

Por definición (integración arbitraria) se tiene:

$$
{ }_{a} I_{x}^{\frac{1}{3}} f(x)=\frac{1}{\Gamma\left(\frac{3}{2}\right)} \int_{a}^{\alpha}(x-s)^{\frac{3}{2}-1} f(s) d s
$$

Generalización de la integral lásica a integral de orden arbitrario

$$
\begin{aligned}
& =\frac{1}{\Gamma\left(\frac{3}{2}\right)^{\alpha}} \int_{x}^{\alpha}(x-s)^{\frac{1}{2}}(2) d s \\
& \left({ }_{a} I_{x}^{\alpha} f\right)(x)=\frac{1}{\Gamma(\alpha)} \int_{a}^{x}(x-t)^{\alpha-1} f(t) d t \\
& =\frac{1}{\Gamma(\alpha)} \int_{a}^{x}(x-t)^{\alpha-1} k d t
\end{aligned}
$$

luego, hacemos cambio de variable:

$$
\text { (1.6) }\left\{\begin{array}{c}
u=x-t \\
t \rightarrow x \Rightarrow u \rightarrow 0 \\
t \rightarrow a \Rightarrow u \rightarrow x-a \\
d t=-d t
\end{array}\right.
$$

Reemplazando (1.5) en (1.6) se tiene:

Luego, haciendo cambio de variable:

$$
(1.4)\left\{\begin{aligned}
u & =x-s \\
\Rightarrow & \Rightarrow x=x-s \\
s \rightarrow a & \Rightarrow u \rightarrow 0 \\
d s & =-d u
\end{aligned}\right.
$$

Reemplazando (1.3) en (1.4) y utilizando la Tabla 1 se obtiene:

$$
\begin{aligned}
I_{a}^{\frac{3}{2}}(2) & =\frac{2}{\sqrt{\pi}} \int_{x-a}^{0} u^{\frac{1}{2}}(d u) \\
& =\frac{-12}{\sqrt{\pi}}\left[\frac{2}{3}\left(u^{\frac{3}{2}}\right)\right]_{0}^{x-a} \\
& =\frac{-4}{3 \sqrt{\pi}}(x-a)^{\frac{3}{2}}
\end{aligned}
$$




\section{Integral arbitraria de la función constante:}

Proposición (Integral arbitraria de la función constante) Si $f: \mathbb{R} \rightarrow \mathbb{R}$ es una función tal que $f(x)=k$ entonces

$$
\left({ }_{a} I_{x}^{\alpha} f\right)(x)=\frac{k}{\Gamma(\alpha+1)}(x-a)^{\alpha}
$$

\section{Prueba}

Por definición de la integral de orden arbitrario, se tiene:

$$
\begin{aligned}
\left({ }_{a} I_{x}^{\alpha} f\right)(x) & =\frac{k}{\Gamma(\alpha)} \int_{x-a}^{0} u^{\alpha-1}(-d u) \\
& =\frac{k}{\Gamma(\alpha)} \int_{0}^{x-a} u^{\alpha-1}(d u) \\
& =\frac{k}{\Gamma(\alpha)}\left[\left.\frac{u^{\alpha}}{\alpha}\right|_{u=0} ^{u=x-a}\right. \\
& =\frac{k}{\Gamma(\alpha+1)}(x-a)^{\alpha}
\end{aligned}
$$

Por lo tanto $\left({ }_{a} I_{x}^{\alpha} f\right)(x)=\frac{k}{\Gamma(\alpha+1)}(x-a)^{\alpha}$

Ejemplo (Integral arbitraria de la función constante) Calcular la integral de orden arbitrario $\alpha=\frac{2}{3}$ y $a=2$ de $f(x)=5$

\section{Solución}

Por la proposición tenemos:

$$
\begin{aligned}
I_{x}^{\alpha} k & =\frac{k}{\Gamma(\alpha+1)}(x-a)^{\alpha} \\
& =\frac{1}{\Gamma\left(\frac{2}{3}+1\right)}(x-2)^{\frac{2}{3}} \\
& =\frac{1}{\Gamma\left(\frac{5}{3}\right)^{\frac{2}{3}}}(x-2)^{\frac{2}{3}} \\
& =\frac{(x-2)^{\frac{2}{3}}}{0.9027}
\end{aligned}
$$

Por lo tanto $I^{\frac{2}{3}}(5)=\frac{(x-2)^{\frac{2}{3}}}{0.9027}$

Integral arbitraria de la función potencia:

$$
\begin{aligned}
& { }_{a} I_{x}^{\alpha}(x-a)^{\beta}=\frac{1}{\Gamma(\alpha)} \int_{0}^{1}[(x-a)(1-t)]^{\alpha-1}[t(x-a)](x-a) d t \\
& =\frac{1}{\Gamma(\alpha)} \int_{0}^{1} t^{(\beta+1)-1}(x-a)^{\alpha-1}(x-a)^{\beta}(x-a) d t \\
& =\frac{(x-a)^{\alpha+\beta}}{\Gamma(\alpha)} \int_{0}^{1} t^{(\beta+1)-1}(1-t)^{\alpha-1} d t
\end{aligned}
$$

Luego por la definición de la función Beta se tiene:

$$
{ }_{a} I_{x}^{\alpha}(x-a)^{\beta}=\frac{(x-a)^{\alpha+\beta}}{\Gamma(\alpha)} B(\beta+1, \alpha)
$$

Por la propiedad de la función Beta:

$$
B(\beta+1, \alpha)=\frac{\Gamma(\beta+1) \Gamma(\alpha)}{\Gamma(\beta+1+\alpha)}
$$

Por lo tanto

\section{Proposición (Integral arbitraria de la función} potencia)

Si $f(x)=(x-a)^{\beta}, \beta>0, x>\alpha$ entonces:

$$
{ }_{a} I_{x}^{\alpha}(x-a)^{\beta}=\frac{\Gamma(\beta+1)}{\Gamma(\alpha+\beta+1)}(x-a)^{\alpha+\beta}
$$

\section{Prueba}

Por la definición de la integral fraccionaria se tiene:

$$
{ }_{a} I_{x}^{\alpha}(x-a)^{\beta}=\frac{1}{\Gamma(\alpha)} \int_{a}^{x}(x-s)^{\alpha-1}(s-a)^{\beta} d s
$$

luego, haciendo un cambio de variable:

$$
(1.8)\left\{\begin{array}{c}
s=a+t(x-a) \\
s-a=t(x-a) \\
s-a=t x-t a \\
a-s=t a-t x \\
a-s+x-a=x-a+t a-t x \\
x-s=(x-a)(1-t) \\
s \rightarrow x \Rightarrow t \rightarrow 1 \\
s \rightarrow a \Rightarrow t \rightarrow 0 \\
d s=(x-a) d t
\end{array}\right.
$$

Reemplazando (1.8) en (1.7) se tiene: 


$$
\begin{aligned}
{ }_{a} I_{x}^{\alpha}(x-a)^{\beta} & =\frac{(x-a)^{\alpha+\beta}}{\Gamma(\alpha)} \cdot \frac{\Gamma(\beta-1) \Gamma(x)}{\Gamma(\beta+1+\alpha)} \\
& =\frac{\Gamma(\beta+1)}{\Gamma(\beta+1+\alpha)}(x-a)^{\alpha+\beta} ; \alpha, \beta>0, x>a
\end{aligned}
$$

\section{Ejemplo (Integral arbitraria de la función}

\section{potencia)}

Calcular la integral arbitraria de orden $\alpha=\frac{1}{3}$ y

$a=4$ de $f(x)=(x-4)^{\frac{1}{2}}$

\section{Solución}

De la proposición (potencial arbitraria)

$$
\begin{aligned}
& { }_{a} I_{x}^{\alpha}(x-a)^{\beta}=\frac{\Gamma(\beta+1)}{\Gamma(\alpha+\beta+1)}(x-a)^{\alpha+\beta}, \alpha, \beta>0, x>a \\
& { }_{4} I_{x}^{\frac{1}{3}}(x-4)^{\frac{1}{2}}=\frac{\Gamma\left(\frac{3}{2}\right)}{\Gamma\left(\frac{11}{6}\right)}(x-)^{-} 4^{6}{ }^{5}
\end{aligned}
$$

Por la propiedad de la función Gamma y la Tabla 1 se obtienen:

$$
{ }_{4} I_{x}^{\frac{1}{3}}(x-4)^{\frac{1}{2}}=\frac{\sqrt{\pi}}{2(0,9407)}(x-4)^{\frac{5}{6}}
$$

\section{Integración arbitraria de la función logaritmo:}

\section{Proposición (Integral de orden arbitrario de la} función logaritmo)

Generalización de la integral clásica a integral de orden arbitrario

Reemplazando (1.10) en (1.9) se tiene:

$$
\begin{gathered}
a_{a}^{I_{x}^{\alpha}} \ln (x-a)=\frac{1}{\Gamma(\alpha)} \int_{0}^{1}[(x-a)(1-t)]^{\alpha-1} \ln [t(x-a)](x-a) d t \\
=\ln (x-a) \int_{0}^{1}(1-t)^{\alpha-1} d t+\frac{(x-a)^{\alpha}}{\Gamma(\alpha)} \int(1-t) \ln (t) d t
\end{gathered}
$$

Haciendo cambio de variable en la segunda integral:

$$
(1.12)\left\{\begin{array}{c}
u=1-t \\
t=1-u \\
t \rightarrow 1 \Rightarrow u \rightarrow 0 \\
t \rightarrow 0 \Rightarrow u \rightarrow 1 \\
d t=-d u
\end{array}\right.
$$

Reemplazando (1.12) en (1.11)

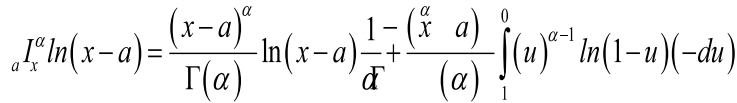

$$
\begin{aligned}
& =\frac{(x-a)^{\alpha}}{\alpha \Gamma(\alpha)} \ln (x-a)-\frac{\left(x^{a} a\right.}{\Gamma(\alpha)} \int_{0}^{1}(u)^{\alpha-1} \ln (1-u) d u
\end{aligned}
$$

Si $f(x)=\ln (x-a)$ tal que $x>a$ entonces

$$
{ }_{a} I_{x}^{\alpha} \ln (x-a)^{\beta}=\frac{(x-a)^{\alpha}}{\Gamma(\alpha+1)}[\ln (x-a)-\gamma-\psi(\alpha+])
$$

\section{Prueba}

De la definición de la integral de orden arbitrario se tiene:

${ }_{a} I_{x}^{\alpha} \ln (x-a)=\frac{1}{\Gamma(\alpha)} \int_{a}^{x}(x-a)^{\alpha-1} \ln (s-a) d s$

luego, haciendo cambio de variable

$$
s=a+t(x-a)
$$

\section{Donde seobtiene:}

$$
\begin{gathered}
s-a=t(x-a) \\
a-s=t a-t x \\
a-s+x-a=x-a+t a-t x \\
x-s=(x-a)(1-t) \\
s \rightarrow x \Rightarrow t \rightarrow 1 \\
s \rightarrow a \Rightarrow t \rightarrow 0
\end{gathered}
$$

luego, se sabe que $d\left(1-u^{\alpha}\right)=-\alpha u^{\alpha-1} d u$

entonces $u^{\alpha-1} d u=-\frac{d\left(1-u^{\alpha}\right)}{\alpha} \mathrm{y}$

reemplazando en la ecuación se tiene

$$
\begin{aligned}
& { }_{a} I_{x}^{\alpha} \ln (x-a)=\frac{(x-a)^{\alpha}}{\Gamma(\alpha+1)} \ln (x-a)+\frac{\left(x \stackrel{\alpha}{a}^{\alpha}\right)}{\alpha \operatorname{La}()} \int_{0}^{1} \ln (1-u) d(1-u) \\
& =\frac{(x-a)^{\alpha}}{\Gamma(\alpha+1)} \ln (x-a)+\frac{(x \stackrel{\alpha}{a})}{\Gamma \alpha+1)}\left[\left(\left(1-u^{\alpha}\right) \ln (1-u)\right)_{0}^{1}+\int_{0}^{1} \frac{1-u^{\alpha}}{1-u} d u\right. \\
& =\frac{(x-a)^{\alpha}}{\Gamma(\alpha+1)}\left[\ln (x-a)+\left(1-u^{\alpha}\right) \ln (1-u)\right]_{0}^{1}+\int_{0}^{1} \frac{1-u^{\alpha}}{1-u} d u
\end{aligned}
$$


Aplicando la propiedad de la función Digamma:

$$
{ }_{a} I_{x}^{\alpha} \ln (x-a)=\frac{(x-a)^{\alpha}}{\Gamma(\alpha+1)}[\ln (x-a)-\gamma-\psi(\alpha+] 1)
$$

\section{Ejemplo (Integral de orden arbitrario logarítmico)}

Calcular la integral fraccionaria de orden $\alpha=\frac{1}{3}$

de $f(x)=\ln (x-2)$

\section{Solución}

De la proposición de la integral arbitraria del logaritmo se tiene

$$
\begin{aligned}
& { }_{a} I_{x}^{\alpha} \ln (x-a)=\frac{(x-a)^{\alpha}}{\Gamma(\alpha+1)}[\ln (x-a)-\gamma-\psi(\alpha+]) \\
& { }_{a}^{I_{x}^{\frac{1}{3}}} \ln (x-2)=\frac{(x-2)^{\frac{1}{3}}}{\Gamma\left(\frac{1}{3}+1\right)}\left[\ln (x-2)-\gamma-\psi\left(\frac{1}{3}+1\right)\right.
\end{aligned}
$$

Por las propiedades de la función Gamma y la Tabla 1 y 2 se tiene:

$$
\begin{aligned}
& =\frac{(x-2)^{\frac{1}{3}}}{\Gamma\left(\frac{4}{3}\right)}\left[\ln (x-2)-\gamma-\left(\psi\left(\frac{1}{3}\right)+\frac{1}{\frac{1}{3}}\right) \mid\right] \\
& =\frac{(x-2)^{\frac{1}{3}}}{\Gamma\left(\frac{4}{3}\right)}\left[\ln (x-2)-\gamma-\left(-\gamma-\frac{\pi}{2 \sqrt{3}}-\frac{3}{2} \ln (3)+\beta\right]\right. \\
& =\frac{(x-2)^{\frac{1}{3}}}{0.8430}\left[\ln (x-2)+\frac{\pi}{2 \sqrt{3}}+\frac{3}{2} \ln (3)-3 \mid\right]
\end{aligned}
$$

Por lo tanto:

$$
{ }_{a} I_{x}^{\alpha} \ln (x-2)=\frac{(x-2)^{\frac{1}{3}}}{0.8430}\left[\ln (x-2)+\frac{\pi}{2 \sqrt{3}}+\frac{3}{2} \ln (3)\right]-3
$$

\section{CONCLUSIONES}

La definición de la integral de orden arbitrario es una generalización de la integral ordinaria o entera.

No existe una única integral arbitraria, sino varias definiciones con diferentes propiedades, cada una de estasdefiniciones exige sus propias condiciones de las funciones que se aplican. En nuestro caso hemos aplicado la definición de Riemann-Liouville.

\section{REFERENCIAS BIBLIOGRÁFICAS}

ARAFET, P., Dominguez A. y Chang, F. (2008). Una introducción al cálculo fraccionario. Cuba: Universidad del Oriente.

BELL, W. (1968). Special functions for Scientists and Engineers. D. Van Nostrand Company Ltd.

Bertram, R. (1975). Fractional calculus and its applications. First. Germany: N.p.

BONILLA, Kilbas, A., and Trujillo, J. (2003). Cálculo fraccionario y ecuaciones diferenciales fraccionarias. Edit. UNED: Madrid.

Chaudhry, M. A.,\& Zubair, S. M. (2002). On a class of incomplete gamma functions with applications. United States of America: Academic Press, INC

GUERRA, J. and Kalla, S. (1990). Aplicaciones del cálculo fraccional para resolver ecuaciones diferenciales. Rev. Téc. Ing. Univ. Zulia 13 (1).1:6.Levedev, N. (1972). Special functions and their applications. First. London: PrenticeHall, Inc.

Miller, K. and Ross, B. (1993).An introduction to the fractional calculus and fractional differential equations. Wiley and Sons: New York

ORTIGUEIRA, Manuel. (2011). Fractional calculus for scientists and engineers. 84th ed. Portugal: Springer.

OLDHAM, K. and Spanier, J. (1974). The fractional calculus: Theory and applications of differentiation and integration to arbitrary order. Ed. Richard Bellman. 111th ed. United States of America: Academic Press, INC.

SANTANU, D. (2011). Functional fractional calculus. Second. India: Springer.

Zill, D. and Warren, W. (2011). Cálculo de varias variables. $4^{\mathrm{a}}$ ed. China: McGraw Hill.

VPO 